\title{
La solución de Wittgenstein al problema del "concepto caballo", o de cómo hablar acerca de la estructura del lenguaje según el Tractatus*
}

Recibido: octubre 17 de 2017 | Aceptado: febrero 23 de 2018

DOI: 10.17230/co-herencia.15.29.6

\author{
Víctor Hugo Chica Pérez* \\ vchica1@gmail.com
}

Resumen El artículo propone interpretar la distinción que ofrece Wittgenstein en el Tractatus entre conceptos ordinarios y conceptos formales como una respuesta a las dificultades que se originan en la distinción fregeana entre concepto y objeto, a las cuales la tradición se ha referido como las dificultades del "concepto caballo". Se mostrará que las expresiones contradictorias y paradójicas que surgen tanto del ataque a dicha distinción (v. g. "el concepto caballo es un objeto"), como de su defensa (v. g. "el concepto caballo no es un concepto"), tienen su origen en la pretensión ilegítima de describir o caracterizar la estructura lógica de la proposición usando para ello otras proposiciones. La distinción entre conceptos ordinarios y formales permite aclarar, primero, en qué sentido esas expresiones son absurdos; segundo, que la distinción entre concepto y objeto solo puede reconocerse en el simbolismo por las características de los signos, pero no puede constituir objeto de descripción.

\section{Palabras clave:}

Concepto caballo, concepto formal, Tractatus, conceptos lógicos.

Wittgenstein's solution to the problem of the "concept horse", or how to talk about the structure of the language according to the Tractatus

\footnotetext{
Abstract This article asserts that the distinction made by Wittgenstein in the Tractatus between 'ordinary concepts' and 'formal concepts' may be construed as a response to the difficulties arising from the Fregean distinction between concept and object, which are traditionally referred to as the 'concept horse problem'. The paper will show that contradictory and paradoxical expressions that arise both from
}

\begin{abstract}
El presente artículo es producto del proyecto de investigación "Lenguaje sin significado: estudio sobre el rol y la evolución del concepto de uso en la obra de L. Wittgenstein", adscrito al grupo de investigación Conocimiento, filosofía, ciencia, historia y sociedad del Instituto de Filosofía de la Universidad de Antioquia y fue financiado por el Comité para el Desarrollo de la Investigación (CODI) de la misma universidad.

** Profesor del Instituto de Filosofía de la Universidad de Antioquia, Medellín, Colombia. OCRID ID: 0000-00025448-0308
\end{abstract}


the attack to the Fregean distinction (e.g., the concept horse is an object) and from its defense (e.g., the concept horse is not a concept), are rooted in an illegitimate attempt, namely, to describe or characterize the logical structure of the proposition by using other propositions. The distinction between ordinary and formal concepts makes it possible to clarify, first, in what sense these expressions are nonsensical; and second, that the distinction between concept and object can only be recognized in symbolism by the characteristics of signs, but it cannot be the object of description.

\section{Keywords:}

Concept horse, formal concept, Tractatus, logical concepts.

\section{Orígenes del problema del 'concepto caballo'}

El problema acerca del 'concepto caballo' tiene su origen en el famoso artículo de Frege "Sobre concepto y objeto" (1974), que aparece en 1892, donde el autor ofrece una serie de aclaraciones a propósito de la crítica que realiza Benno Kerry a la distinción entre concepto y objeto. Es bien sabido que Frege analiza la estructura de la proposición distinguiendo dos tipos de componentes: el concepto y el objeto. Toda proposición está compuesta, según el argumento de Frege, por una parte en sí misma completa o saturada, el objeto o sujeto lógico, y una parte incompleta o insaturada, el concepto. Así en la proposición "Bucéfalus es un caballo" tenemos que 'Bucéfalus' es la parte completa, el objeto, y 'es un caballo' la parte insaturada, el concepto. Según Kerry dicha distinción no puede mantenerse, como Frege pretende, en un caso como "el concepto caballo es un concepto fácil de adquirir", pues aquí, según Kerry, la expresión 'el concepto caballo' cumple con la condición fundamental que Frege exige de todo objeto, y es la de caer bajo un concepto, en este caso bajo el concepto 'fácil de adquirir'; de esta manera, según Kerry, 'el concepto caballo' se vuelve un objeto y se desvanece así la diferencia entre concepto y objeto.

Para enfrentar el desafío que plantea el análisis de Kerry, Frege advierte, primero, que el uso que él hace de la palabra 'concepto' es un uso estrictamente lógico; segundo, que si bien no es posible dar una definición de qué sea un concepto, dada su simplicidad lógica, lo que él va a hacer es instruir al lector a través de señas o aclaraciones, para que entienda lo que ha querido decir con la 
palabra 'concepto'. Que Frege haga un uso estrictamente lógico de la palabra 'concepto' quiere decir que dicho término está en función de caracterizar un tipo de expresiones, precisamente aquellas que exhiben un carácter incompleto o insaturado, que se reconoce fácilmente cuando la expresión se separa de los demás componentes de la proposición, por ejemplo sustituyendo en la proposición el nombre propio por una variable. Por tanto expresiones como 'ser verde' o 'ser mamífero', que funcionan como predicados gramaticales en afirmaciones del tipo 'el árbol del patio es verde' o 'ese caballo es mamífero', poseen claramente ese carácter insaturado o incompleto que se hace evidente si las separamos del resto de la proposición, como se ven en: ' $\mathrm{x})$ es verde' y '( $\mathrm{x})$ es mamífero'. Y en eso consiste su carácter estrictamente predicativo, en el hecho de necesitar ser completadas para expresar algo con sentido. Las otras dos expresiones: 'el árbol del patio' y 'ese caballo', funcionan como nombres propios cuya tarea es designar un objeto.

Ahora, para un objeto es imposible funcionar lógicamente como predicado, incluso, en aquellos casos donde aparecen como predicados gramaticales, por ejemplo en proposiciones del tipo "la estrella de la mañana es Venus” o "el maestro de Platón fue Sócrates”. En estos casos tanto 'Venus' como 'Sócrates' son nombres propios y el 'es' opera aquí como signo de igualdad indicando que dichos nombres pueden intercambiarse respectivamente con los nombres: 'la estrella de la mañana' y ‘el maestro de Platón', los cuales designan los mismos objetos que 'Venus' y 'Sócrates' respectivamente.

A diferencia de los ejemplos precedentes, donde el 'es' funciona como cópula, esto es, como parte de la expresión conceptual, la operación que introduce el 'es' cuando se usa como signo de igualdad es una relación convertible, por eso las dos expresiones que componen la proposición pueden intercambiarse sin alterar su semántica. No sucede lo mismo cuando el 'es' funciona como cópula pues la relación

1 Kelly Dean (2007) argumenta que los malentendidos de Kerry son malentendidos inducidos por la forma como Frege se expresa en los Las leyes fundamentales de la aritmética, pues mientras Frege ofrece lo que él mismo denomina 'pistas' o 'claves' para entender las nociones lógicamente simples, que no tienen definición posible, Kerry reclama precisamente una definición que aclare el contenido de dichas nociones, y según Dean, Kerry toma las observaciones de Frege como definiciones imprecisas (cfr. Dean, 2007). 
de caer un objeto bajo un concepto no es una relación convertible; por eso no es posible formar una proposición como 'ser verde es el árbol del patio' o 'ser mamífero es ese caballo'. Frege ofrece una paráfrasis que ayuda a aclarar la condición saturada o completa de las expresiones que funcionan como nombres propios: "la estrella de la mañana no es otra cosa que Venus" o "el maestro de Platón no es otro que Sócrates”. Aquí se aprecia que 'Venus' y 'Sócrates' hacen parte de un predicado gramatical, el de 'no ser otra cosa que $x$ ' pero no constituyen ellas mismas un predicado, o dicho de forma más clara, no tienen carácter predicativo, puesto que no son expresiones incompletas. Por tal razón el significado de las palabras 'Venus' y 'Sócrates' solo puede introducirse como objeto en una proposición, nunca como concepto.

Para aclarar el carácter predicativo de los conceptos Frege recurre nuevamente a la paráfrasis: "Bucéfalus es un caballo" puede reescribirse como "Bucéfalus cae bajo el concepto caballo". Aquí se advierte claramente que el predicado no es solo la combinación de palabras 'el concepto caballo', ésta es solo una parte del predicado que es 'cayendo bajo el concepto caballo', y que significa lo mismo que 'un caballo' o con mayor precisión 'es un caballo'. Esto confirma, según Frege, que es imposible borrar la diferencia entre concepto y objeto, pues ambos exhiben propiedades lógicas distintas o, lo que es lo mismo, muestran características funcionales diferentes.

Con estas cosas claras, Frege confronta la crítica de Kerry sobre la distinción entre concepto y objeto atacando la forma como el autor asigna la condición de concepto a la expresión 'el concepto caballo'. Frege aclara que un concepto no puede desempeñarse como objeto, esto es, como nombre propio y agrega que quien entiende la serie de palabras el concepto 'caballo' como un concepto, está usando la noción de concepto de manera totalmente distinta a la suya, no en un sentido estrictamente lógico, sino tal vez en un sentido metafísico o psicológico. El argumento de Frege se apoya en determinadas distinciones lingüísticas que permiten constatar las diferencias entre los dos tipos lógicos que componen la proposición. Frege argumenta que "con el singular del artículo determinado se indica siempre a un objeto, mientras que el artículo indeterminado acompaña a una palabra-concepto" (1974, p. 62). Por eso la expresión 'el concepto caballo' no puede ser un concepto, ya que carece del carácter 
incompleto o insaturado que caracteriza los conceptos genuinos, como 'ser verde', 'ser un hombre' o 'ser un caballo'. Según Frege las tres primeras palabras 'el concepto caballo' se comportan como un nombre propio, e igual que 'Venus' y 'Sócrates', no se pueden usar predicativamente.

Aquí tiene lugar una extraña situación relativa a la idea de que "el concepto caballo no es ningún concepto" la cual tiene su origen, según Frege, en una inevitable dificultad lingüística que surge cuando intentamos afirmar algo acerca de un concepto. Que aquí nos encontramos en una situación especial se aprecia en el hecho de que se recurre a cierta clase de marcas especiales para llamar la atención sobre el uso peculiar de la palabra caballo, como son las comillas en Kerry o las letras cursivas que usa Frege. Esto, para Frege, se debe a que en las investigaciones lógicas se tiene, frecuentemente, la necesidad de enunciar algo acerca de un concepto y, en esos casos, nos vemos inclinados a esperar que en dicho enunciado el concepto aparezca como el sujeto gramatical, pero esto es imposible. Por su naturaleza predicativa un concepto no puede aparecer como sujeto gramatical, por eso, en palabras de Frege, el concepto "tiene primero que transformarse en un objeto, o para hablar más exactamente, tiene que ser representado por un objeto" (1974, p. 64). Esto se logra anteponiendo las palabras 'el concepto', para designar ese objeto que "representa" el concepto del cual se quiere dar a entender algo. Este argumento evidencia la dificultad con la cual nos encontramos a la hora de querer decir algo sobre un concepto, y si bien Frege es consciente de esa dificultad la solución que ofrece no es completamente satisfactoria pues afirmar que un concepto debe transformarse en un objeto, o ser representado por uno, para poder hacer alguna aclaración sobre él, genera más confusiones, lo cual pone en riesgo la diferencia que el mismo Frege proclama entre concepto y objeto. ${ }^{2}$

2 Anthony Kenny advierte correctamente que el argumento de Frege está orientado a mostrar que expresiones del tipo 'el concepto caballo es fácil de adquirir' no constituyen contraejemplos que pongan en riesgo la distinción entre concepto y objeto ( $c f r$. Kenny, 1995, pp. 122-125). Sin embargo, lo que Kenny no advierte es que el mismo Frege no puede ofrecer un análisis satisfactorio de la situación conceptual que tiene lugar en expresiones como 'el concepto caballo no es ningún concepto' o 'el concepto caballo no es ningún objeto’. Estas expresiones no son análogas a la anterior, y lo que evidencian es la dificultad inherente a la distinción misma entre concepto y objeto. 


\section{La solución del Tractatus a las dificultades del 'concepto caballo'}

El Tractatus Logico-Philosophicus ${ }^{3}$ (2009) vio la luz por primera vez en 1919. Allí el problema de la distinción entre concepto y objeto es parte de una amplia discusión con Frege y Bertrand Russell acerca de la naturaleza de la proposición y del simbolismo de la lógica cuyo objetivo es resolver las dificultades propias de la empresa fregeana que consiste en aclarar los rasgos lógicos o estructurales de la proposición. Sin embargo, aquí nos centraremos únicamente en los aspectos conceptuales necesarios para entender la manera como el TLP se enfrenta al problema del 'concepto caballo'. Lo único que no debe perderse de vista es que Wittgenstein concibe en el TLP la proposición como una figura: "La proposición es una figura de la realidad. La proposición es un modelo de la realidad tal como la pensamos" (TLP, 4.01). Pero tal figura es puramente lógica, es decir, la proposición representa una situación o un hecho a través de la forma como en ella se combinan sus elementos, o en otras palabras, a través de su propia estructura que la capacita para efectuar dicha representación.

En el marco de esta concepción figurativa de la proposición tiene lugar una discusión inédita hasta el momento en la tradición filosófica clásica relativa a la naturaleza de las nociones y expresiones que usamos en filosofía para hablar acerca del lenguaje. Wittgenstein sugiere que dichas nociones gozan de una condición especial, la cual puede aclararse gracias a una diferencia que la concepción figurativa permite construir entre lo que el lenguaje describe y lo que el lenguaje muestra en sí mismo. A continuación se aclarará esta distinción junto con el aparato conceptual básico que nos permitirá solucionar, desde la lógica del TLP, las dificultades que plantea el problema del 'concepto caballo'.

\section{Lo que el lenguaje dice y lo que el lenguaje muestra}

La distinción entre lo que el lenguaje dice y lo que él mismo muestra es una consecuencia directa de la concepción figurativa de

3 De aquí en adelante TLP. 
la proposición. ${ }^{4} \mathrm{Si}$ se entiende la proposición como una figura lógica de una situación y se admite que el fundamento de dicha condición figurativa es que la proposición posee rasgos estructurales específicos a través de los cuales puede coordinarse con la situación que representa se tiene, por un lado, el sentido de la proposición, lo que la proposición representa y, por otro, su forma lógica o estructura, lo que la capacita para expresar dicho sentido. Wittgenstein es claro al estipular que ninguna figura "puede figurar su forma de figuración" (TLP, 2.172) y, en consecuencia, una proposición "no puede representar [darstellen] la forma lógica; ésta se refleja [spiegelt] en ella" (TLP, 4.121). De ahí que toda proposición que represente una situación, sea efectiva o posible, refleja o exhibe su forma o estructura.

Esta distinción introduce una restricción y es la imposibilidad de construir una proposición que describa o represente la forma lógica de cualquier otra proposición, pues la forma lógica es justo lo que caracteriza un conjunto arbitrario de signos como proposición. Con esta distinción Wittgenstein enfrenta una dificultad que Frege ya advertía desde que escribe la Conceptografía (1879) y es la que surge a la hora de intentar aclarar las propiedades lógicas de la proposición: el límite con el que choca nuestro lenguaje cuando intentamos explicar su estructura. Esta es la dificultad que se manifiesta en afirmaciones tales como "el concepto caballo no es ningún objeto" o "el concepto caballo no es ningún concepto", donde pareciera que nuestros modos de expresión son insuficientes para describir claramente, por ejemplo, las propiedades de un concepto.

Sin embargo desde la perspectiva del TLP no se trata de una insuficiencia de nuestro lenguaje, se trata es de que las propiedades estructurales de la proposición son indecibles: "La proposición puede representar la realidad entera, pero no puede representar lo que ha de tener de común con la realidad para poder representarla- la forma lógica" (TLP, 4.12). Que no es posible describir la forma

4 Distinción fundamental en el TLP según lo expresó el propio Wittgenstein: "El punto principal es la teoría de lo que puede expresarse (gesagt) por las proposiciones, esto es, por el lenguaje [...] y lo que no puede ser expresado por proposiciones, sino sólo mostrado (gezeigt); creo que este es el problema cardinal de la filosofía" (Carta 37 a Russell, 18-081919; Wittgenstein, 1979, p. 68).

5 John Koethe (1996, p. 34 y ss.) hace el experimento de intentar describir la estructura de una proposición como 'John ama a María', para finalmente reconocer el fracaso 
lógica de la proposición se explica porque cualquier intento de figurar la estructura de una proposición exigiría representar lo que tienen en común la proposición y la situación, esto es, la forma lógica; pero cualquier proposición exhibirá la misma forma lógica, es decir, tendría inevitablemente identidad de forma con respecto a la primera proposición y a la realidad, no tendría por tanto éxito en la descripción de eso que tienen en común la situación y la proposición. ${ }^{6}$ De ahí que la única alternativa sería poder "situarnos con la proposición fuera de la lógica" (TLP, 4.12), pero construir un lenguaje por fuera de la lógica constituye claramente un absurdo, pues lo esencial a todo lenguaje, según la concepción figurativa, es poder representar una situación posible o expresar un sentido, y esto presupone una forma lógica, una estructura.

Dado entonces que "lo que en el lenguaje se refleja, nosotros no podemos expresarlo por el lenguaje" (TLP, 4.121) debe admitirse que nuestro acceso a la forma lógica de una proposición solo es posible a través de la misma proposición que la exhibe, reconociendo las propiedades lógico-sintácticas de los componentes del signo proposicional cuando es puesto en relación con un estado de cosas; solo en tal circunstancia se nos revela la lógica de la proposición. Esto es justamente lo que Wittgenstein quiere vindicar en el TLP: que la lógica del lenguaje se muestra en el uso del signo proposicional, o lo que es lo mismo, en la práctica misma del lenguaje cuando se asevera una situación o cuando se construyen relaciones entre proposiciones

de tal intento: supongamos que intentamos representar esa proposición o decir que los nombres "Juan" y "María" estaban dispuestos en ese orden. Podríamos hacerlo (de acuerdo con la teoría figurativa) solo organizando y mostrando los nombres (de los nombres originales) "Juan" y "María" en una forma lógica que es la misma en que los nombres están dispuestos en la proposición original "Juan ama a María", pero esa es solo la forma pictórica del estado de cosas de Juan amando a María; y en virtud de compartir esa forma, la proposición original dice que Juan ama a María. Así, parecería que nuestra nueva proposición, si dice o representa realmente algo, debe decir que (el nombre) 'Juan' ama (el nombre) 'María' o representa el estado de cosas de 'Juan' amando a 'María', lo cual es un sinsentido (asumiendo que la ontología del Tractatus es categórica y que amar no es una relación posible entre elementos lingüísticos), o al menos nada en absoluto como lo que estábamos tratando de decir (1996, p. 36).

6 Una reflexión interesante al respecto aparece en los Diarios (29.5.15 [2009]), pero el problema allí no se desarrolla, solo se formula la pregunta de por qué no sería posible un modo de expresión mediante el que me sea posible hablar acerca del lenguaje, de tal modo que éste pueda aparecer como algo coordinado con otra cosa. 
para inferir, argumentar, etcétera. En este sentido debe entenderse la observación de Wittgenstein:

Así, una proposición "fa" muestra que en su sentido aparece el objeto $a$; dos proposiciones "fa" y "ga", que en ambas se habla del mismo objeto. El que dos proposiciones se contradigan entre sí lo muestra su estructura; de igual modo, el que una se siga de la otra. Etcétera. (TLP, 4.1211)

Es decir, cualquiera que entienda el sentido de las proposiciones "Sócrates es filósofo" y "Sócrates es griego" puede ver que ambas hablan acerca del mismo objeto, 'Sócrates', o que ambas dicen propiedades distintas del mismo objeto, etcétera. Solo en la práctica del lenguaje tiene lugar la lógica de la proposición. En este sentido McGinn comenta acertadamente:

Lo que Wittgenstein nos está haciendo ver gradualmente es que la forma lógica [...] se manifiesta en la manera en que las expresiones se usan con sentido. De la misma manera, nosotros comprendemos lo que es esencial para el sentido de un signo, no teóricamente en la forma de un fragmento de conocimiento proposicional, sino prácticamente en nuestro saber cómo usar un signo con sentido. (2001, p. 28)

Que entendamos la proposición muestra que reconocemos en ella su esencial conexión con la realidad. Aquí se puede formular la cuestión sobre cuál es entonces el tipo de explicación que cabe elaborar con respecto a la estructura de nuestras expresiones y cuál es la naturaleza de las explicaciones de Frege, y del mismo Wittgenstein, acerca de la lógica del lenguaje. Al respecto Wittgenstein introduce una aclaración fundamental:

Podemos hablar, en cierto sentido, de propiedades formales de los objetos y estados de cosas o, respectivamente, de propiedades de la estructura de los hechos y, en el mismo sentido, de relaciones formales y relaciones de estructuras [...]

Pero el darse efectivo de tales propiedades y relaciones internas no puede ser afirmado mediante proposiciones, sino que se muestra en las proposiciones que representan aquellos estados de cosas y que tratan de aquellos objetos. (TLP, 4.122)

Llama aquí la atención que Wittgenstein diga que en cierto sentido se puede hablar de la estructura y de las propiedades formales de los 
hechos, y de los objetos que las proposiciones representan, pero ¿en qué sentido podemos hablar de lo que tienen en común las figuras lógicas y los hechos, esto es, de sus rasgos estructurales, sin violar la restricción que impone el carácter indecible de la forma lógica?

El TLP es categórico en afirmar que la lógica de los hechos y de la proposición es indecible, pero sugiere que es posible introducir aclaraciones sobre cómo el lenguaje cumple su papel de figurar hechos o situaciones, aunque reconoce que dichas aclaraciones constituyen una manera de hablar inusual que nos permite ver, por ejemplo, que hay un límite, y es que el lenguaje no está diseñado para describir su propia estructura; que hay partes que simbolizan de modo y manera distintas, etcétera. Es bastante esclarecedor que Wittgenstein advierta en ese mismo parágrafo que expresiones como 'propiedad formal' o 'propiedad estructural' se introducen estratégicamente para llamar la atención sobre las confusiones que podrían presentarse cuando se quiere aclarar algo acerca del lenguaje sin la precaución de diferenciar claramente entre la herramienta con la cual se está realizando el análisis y aquello que es objeto del análisis. El objeto del análisis es nuestro propio lenguaje ordinario y sus propiedades figurativas, con el cual describimos el mundo de experiencia en general; y la herramienta con la que intentamos analizarlo son cierto tipo de nociones y afirmaciones, las aclaraciones que dan forma al TLP, esto es, observaciones especiales que dan a entender algo acerca de nuestro lenguaje y sus propiedades figurativas.

Lo que me interesa destacar es que cuando Wittgenstein sugiere que en cierto sentido podemos hablar de las propiedades formales de la proposición, puede afirmarse que ese sentido en el que podemos hablar de tales propiedades y relaciones formales es, si cabe la expresión, indirecto, pues no podemos afirmar cuáles sean esas propiedades estructurales; pero sí podemos dar a entender que la proposición posee propiedades estructurales que ella misma exhibe: "La existencia de una propiedad interna de un posible estado de cosas no viene expresada por una proposición, pero se expresa por sí en la proposición que representa el estado de cosas, mediante una propiedad interna de esta proposición" (TLP, 4.124). De igual forma, Wittgenstein afirma que la existencia "de una relación interna entre posibles estados de cosas se expresa lingüísticamente mediante una 
relación interna entre las proposiciones que los representan" (TLP, 4.125). Sin embargo: "Sería tan absurdo [unsinnig] atribuir a la proposición una propiedad formal como negársela” (TLP, 4.124). Pero ¿cómo es posible construir ese tipo de expresiones que constituyen el aparato analítico del TLP? ¿Cuál es la justificación de esa forma inusual de hablar?

\section{Conceptos formales frente a conceptos ordinarios}

Para responder a las cuestiones anteriores se torna central la idea de concepto formal. Wittgenstein afirma que: "En el sentido en el que nosotros hablamos de propiedades formales, podemos hablar ahora también de conceptos formales" (TLP, 4.126). Es decir, de forma análoga a como trazamos la diferencia entre las propiedades estructurales de la proposición y las propiedades en sentido ordinario, las cuales Wittgenstein denomina también externas, podemos trazar la diferencia entre conceptos en sentido ordinario, los que usamos para expresar una propiedad ordinaria o externa, como ser café, ser caballo, etcétera, y conceptos formales, los que expresan una propiedad estructural, ${ }^{7}$ como ser concepto o ser objeto. Wittgenstein es bastante cauteloso en este punto puesto que la misma confusión que se presenta entre propiedades formales y ordinarias, se da también entre conceptos formales y ordinarios. ${ }^{8}$ Por eso es necesario

7 Según Ishiguro (1969) la idea de 'concepto formal' del TLP es análoga a la idea de 'noción lógica' en Frege la cual también se refiere a nociones que están en función del esclarecimiento de la estructura de la proposición (cfr. Ishiguro, 1969).

8 Wolfgang Kienzler (2011) defiende que la importancia de esta noción radica en que la distinción entre conceptos formales y ordinarios constituye el núcleo de todo el TLP (cfr. Kienzler 2011), y que tal distinción debe comprenderse para comprender el sentido de todo el TLP. Alega, además, que éste es el único lugar en todo el texto donde Wittgenstein introduce un vocabulario especial, y donde dice algo acerca de la red conceptual que articula el TLP. Si bien es erróneo afirmar que éste es el único lugar donde Wittgenstein introduce un vocabulario especial, pues conceptos como espacio lógico, forma de figuración, etcétera, son nociones técnicas propiamente tractarianas, sí es correcto que en estos fragmentos Wittgenstein introduce una aclaración fundamental acerca del vocabulario especial que caracteriza todo el TLP: que todos los conceptos fundamentales del TLP son conceptos formales. Wittgenstein ofrece los siguientes ejemplos: 'Lo mismo vale para las palabras 'complejo', 'hecho', 'función', 'número', etc. Todas ellas designan conceptos formales" (TLP, 4.1272). Y claramente podemos agregar otras como 'mundo', 'estados de cosas', 'proposición', 'concepto', etcétera. De tal forma, entender la naturaleza y las características de estos conceptos esclarecerá la naturaleza de las afirmaciones y proposiciones del TLP como sugiere Kienzler. 
definir las características que diferencian los conceptos formales de los ordinarios.

Sobre cuáles son esas características, es posible identificar algunas a partir de los numerales 4.126 y 4.1272 del TLP: 1) Los conceptos formales no pueden ser simbolizados como funciones, como se hace con los conceptos ordinarios o propiamente dichos. 2) Su "definición" es siempre circular. 3) Las afirmaciones en las que aparecen no constituyen proposiciones con sentido. A continuación aclararé cada criterio.

\section{Los conceptos formales no son funciones}

En 4.126 Wittgenstein afirma que: "El concepto formal no puede representarse por una función como se hace con los conceptos propiamente dichos. Por sus características, las propiedades formales no pueden ser expresadas por funciones". La razón es que los conceptos formales expresan las propiedades formales de ciertos símbolos, dicen algo acerca de la manera como se usa un signo en el entramado proposicional, no hablan sobre objetos o cosas en el mundo.

En este sentido, el numeral 4.126 del TLP advierte sobre la confusión entre los conceptos propiamente dichos, que expresan propiedades de cosas, objetos o entes, y los conceptos formales que expresan propiedades de símbolos, esto es, propiedades de carácter formal. Es por esto que constituye un error suponer que expresiones como 'ser objeto', 'ser concepto', 'ser número', etcétera, cualifican una clase de entidades. Este error es comprensible, como bien comenta Hacker, porque: "Tal como estos están representados en los lenguajes naturales, se ven como palabras conceptuales genuinas" (2001, p. 145), es decir, la semejanza entre expresiones como "Bucéfalus es un caballo" y "Bucéfalus es un objeto" nos lleva a considerar que 'objeto' y 'caballo' expresan por igual cualidades de Bucéfalus. Sin embargo, continua Hacker: "Ellos [los conceptos formales] son en efecto nombres de variables, y así deberían estar representados en una notación perspicua" (2001, p. 145); es decir, nociones como objeto, concepto, proposición, etcétera, se expresan en una notación lógica adecuada como variables de algún tipo en el lenguaje sígnico.

Aquí Hacker tiene en mente el caso que expone el mismo Wittgenstein en 4.1272 , que se ha convertido en el paradigma 
del concepto formal, el caso del concepto 'objeto' o 'cosa'. Según el argumento de Wittgenstein en 4.126 un concepto propiamente dicho aparecerá en la notación lógica como una función, así para 'Bucéfalus es un caballo', cuya representación en la notación lógica es Fa, la función 'F' es el símbolo del concepto caballo, mientras que 'a' es el símbolo del objeto Bucéfalus. ${ }^{9}$ De forma semejante en 'hay caballos', que se representa $\mathrm{\Xi x}(\mathrm{Fx})$, la F es, igualmente, el símbolo del concepto o propiedad 'caballo'. ${ }^{10}$

Ahora, con base en esta notación lógica, puede hacerse el siguiente análisis de la proposición "Bucéfalus es un caballo": la proposición contiene una propiedad, que simbolizamos con F, la cual se predica de un objeto que simbolizamos con 'a', en otras palabras, la expresión 'es un caballo' es un concepto y 'Bucéfalus' es un objeto. Pero ésta última afirmación ['Bucéfalus' es un objeto] no ofrece ninguna propiedad que corresponda al significado del signo 'Bucéfalus'; la afirmación no indica ninguna cualidad del caballo llamado Bucéfalus sino que dice algo sobre el rol que desempeña el signo 'Bucéfalus' en la proposición. ${ }^{11}$ A partir de un uso adecuado de la notación lógica se sigue que la afirmación 'Bucéfalus es un objeto' no puede a su vez simbolizarse de ninguna manera pues, según 4.1272, 'ser objeto' no es una propiedad en el mismo sentido en que lo es 'ser caballo'. Correctamente advierte McGinn:

Si no vemos esto claramente, entonces podemos estar tentados a tratar "objeto", "número", "complejo", "hecho" como conceptos genuinos u ordinarios que tienen una extensión, es decir, que coleccionan una clase de cosas por medio de una propiedad, de la misma manera que 'libro' colecciona una clase de cosas por medio de una propiedad. (McGinn, 2006, p. 183)

9 Un argumento análogo puede introducirse para el concepto de 'función', que es también un concepto formal. "Bucéfalus es un caballo" se representa en la notación como Fa, donde 'a' es el símbolo del objeto y 'F' el del concepto propiamente dicho, que es una 'función'. En esta última afirmación es claro que la palabra 'función' es un concepto formal que se muestra en el uso lógico sintáctico del signo ' $F$ ', y no es expresable, ella misma, por medio de una función.

10 Cabe agregar el caso que Wittgenstein menciona, "hay dos objetos que son caballos", o "hay dos caballos", cuya notación correcta sería $\mathrm{g}(\mathrm{x}, \mathrm{y})[\mathrm{Fx}$. Fy]

11 En palabras de Hamlyn (1959), la manera como cada variable representa o exhibe un uso, indica o señala un concepto formal. 
Por eso no es posible formar una expresión en la notación lógica como 'Oa' donde 'O' represente la propiedad de 'ser objeto', pues en la notación ser objeto es un rasgo del símbolo 'a', esto es, de todos aquellos que caen bajo un concepto. De forma análoga, tampoco podría simbolizarse la afirmación de que "caballo es un concepto", pues ser concepto es también un rasgo de ciertos símbolos. De esta manera, que la expresión 'ser caballo' es un concepto se reconoce en el signo cuando opera con sentido en el entramado proposicional, y la notación lógica es solo una ayuda para reconocer, a través de una expresión como Fa -donde la letra F está por 'ser caballo'-, que dicha expresión constituye una propiedad de un objeto. Este criterio es aplicable para todos los conceptos formales, ninguno puede ser tratado como una propiedad y por tanto representado como una función.

Esta característica de los conceptos formales refleja, como sugiere McGinn, que la delimitación wittgensteiniana entre conceptos formales y conceptos propiamente dichos está en función de satisfacer la necesidad que tenemos de aclarar la manera como simbolizan determinados signos, introduciendo para ello expresiones especiales -los conceptos formales-. En cambio los conceptos genuinos dicen algo sobre el significado de los signos, esto es, dicen cómo están las cosas, hablan sobre sus características, cualidades y relaciones. ${ }^{12}$ Que podamos afirmar que 'Bucéfalus' es un objeto, aclara que el signo 'Bucéfalus' simboliza como un nombre propio. Igualmente, que podamos afirmar que 'caballo' es un concepto, aclara que 'ser caballo' opera como una función, la que atribuye, por ejemplo al objeto 'Bucéfalus', todas las propiedades que tienen los caballos; como lo señala McGinn: "los conceptos formales se ocupan de los símbolos y de sus modos de significación, como se manifiesta en su empleo en proposiciones con sentido, y no de lo que significan los símbolos" (2006, p. 183).

\section{El carácter circular de la aclaración de todo concepto formal}

Es posible afirmar que Frege es el primero en advertir la dificultad inherente a la definición o delimitación de los conceptos

12 Cfr. McGinn (2006). 
fundamentales de la lógica cuando señala en "Sobre concepto y objeto" (1974) el problema con el que se encuentra el lógico a la hora de aclarar sus nociones fundamentales, a saber, que no es posible definir el concepto 'concepto' porque éste, así como la noción de 'objeto' es lógicamente simple. De esta forma, según el argumento de Frege, así como sucede con el caso del químico, a quien no se le puede pedir que divida todos los elementos, al lógico tampoco se le puede pedir que defina todos los conceptos, dado que en algunos casos, como los arriba mencionados, tal cosa no puede hacerse.

Tal imposibilidad tiene su fundamento, siguiendo a Frege, en el hecho de que las nociones fundamentales de la lógica dan forma al sistema completo de la lógica, desde el cual alcanza expresión todo pensamiento posible, y suponer que puede darse una definición o una teoría acerca de las nociones fundamentales que dan forma a dicho esquema sería ponerse sobre tal sistema articulando una especie de metalógica, lo que desde el punto de vista de Frege, y de Wittgenstein, es sencillamente absurdo. Las nociones fundamentales de la lógica, como 'concepto' y 'objeto', se presuponen para articular la idea misma de una definición o de una explicación conceptual, en esto consiste justamente que sean básicas, que son presupuesto de cualquier uso con sentido del lenguaje. Esto conduce a Frege a introducir la idea de elucidación, para el caso de los conceptos lógicos, en lugar de la idea de definición. Y así como Frege introduce elucidaciones para explicar los conceptos fundamentales de su Conceptografía, de forma semejante en el TLP la aclaración de los conceptos formales no se introduce mediante definiciones sino mediante elucidaciones. ${ }^{13}$

13 David Hamlyn (1959) afirma que la aclaración de todo concepto formal en el TLP es circular, esto es, que su definición siempre presupone el concepto mismo que se está intentando aclarar. Hamlyn no pretende ofrecer propiamente una interpretación del TLP sino que apela a Wittgenstein para aclarar cómo los conceptos formales conducen a lo que podría denominarse tesis metafísicas, y destaca cómo Wittgenstein mismo, posteriormente en las Investigaciones Filosóficas, ofrece un antídoto contra esta tendencia al señalar que algunos conceptos no tienen límites estrictos, por lo cual su aclaración no puede hacerse por medio de una definición, y este sería el caso de los conceptos formales. No obstante, el autor afirma que Wittgenstein viola este principio y ofrece definiciones de carácter general sobre diversos conceptos formales como 'proposición', 'mundo', 'hecho', etcétera (Hamlyn, 1959). Con todo, considero que en el TLP mismo es consecuente con ese principio de circularidad que caracteriza la aclaración de todo concepto formal, y que Wittgenstein no tiene dificultad alguna en admitir que para aclaración de los conceptos formales, en algún sentido, debemos presuponer los conceptos mismos que estamos intentado aclarar. 
Wittgenstein es consciente en el TLP de esta particularidad de los conceptos lógicos y desarrolla, en cierto sentido, la intuición de Frege de que la forma de aclarar los conceptos fundamentales de la lógica es mostrando, mediante señas y ejemplos, su lugar en el sistema completo de la lógica. Los conceptos formales hablan sobre los símbolos, específicamente sobre el uso lógico sintáctico de los signos, y en lógica la determinación del uso lógico sintáctico de una parte de la proposición presupone el reconocimiento del funcionamiento de todo el mecanismo, de tal forma que la aclaración de un concepto formal presupone todo el sistema conceptual que contiene el concepto mismo que se pretende aclarar. ${ }^{14}$

Este principio de circularidad es vital para enfrentar la dificultad del 'concepto caballo'. Wittgenstein mismo advierte que: "Aunque a la proposición sólo le es dado determinar un lugar del espacio lógico, el espacio lógico total tiene, sin embargo, que venir dado ya por ella" (TLP, 3.42). Es decir, toda la lógica del lenguaje está dada en una sola proposición con sentido, pues una figura lógica cualquiera de una situación contiene ya la forma general de toda proposición posible; este es el sentido de la afirmación según la cual "La proposición atraviesa el espacio lógico entero" que aparece en el mismo numeral 3.42. Esta característica de nuestro lenguaje se refleja además en el hecho de que para aclarar las propiedades formales de un signo, esto es, la manera como éste se desempeña en el entramado de la proposición con sentido, se presuponen las propiedades formales de todas las partes que simbolizan en la proposición, pues según la lógica del TLP solo en el signo proposicional puede estipularse el rol de las expresiones que caracterizan el sentido de la proposición. Esto implica que para explicar la lógica de un símbolo, es decir, sus propiedades formales, se deben conocer las propiedades formales de todos los demás símbolos, por ejemplo, es imposible saber si un signo

14 Es interesante anotar, como bien aclara Dawn Phillips (2007), que en el TLP coexisten de manera simultánea dos tipos de análisis, uno que podríamos llamar análisis elucidatorio, que permite diferenciar las proposiciones con sentido de los sinsentidos, y otro el análisis lógico, que permite reconocer los componentes estructurales de la proposición con sentido (cfr. Phillips, 2007). Cabría precisar la distinción de Phillips aclarando que el análisis elucidatorio es el que se ejecuta a través de las propias proposiciones del TLP, mientras el análisis lógico es el que se lleva a cabo haciendo uso de la notación lógica que el TLP propone, como las tablas de verdad, etcétera. 
simboliza como concepto si no se sabe cuándo un signo funciona como objeto o como operación; el rol de un símbolo siempre es un rol contextual, solo en el entramado del signo proposicional tienen los signos un papel definido en la sintaxis lógica.

Un ejemplo bastante intuitivo de esa circularidad lo constituye la aclaración de la noción misma de concepto ${ }^{15}$ en Frege puesto que para aclarar qué es un concepto es necesario presuponer la idea misma de proposición, a la vez que la noción de proposición presupone, para ser aclarada, la distinción entre concepto y objeto. De forma semejante puede advertirse que en el TLP todos los conceptos que se introducen para aclarar algo sobre nuestro lenguaje y su lógica están interconectados y se explican mutuamente constituyendo el TLP mismo un sistema conceptual claramente sincronizado. De esta manera, por ejemplo, la aclaración del concepto objeto presupone las nociones de hecho o situación, ${ }^{16}$ así como la aclaración de la noción de hecho requiere hacer referencia a la noción de objeto. ${ }^{17} \mathrm{Si}$ una proposición presupone toda la lógica, necesariamente todos los conceptos que se introducen para aclarar las propiedades formales del signo proposicional están interconectados, el mismo TLP es expresión de esa interdependencia de los conceptos fundamentales de la lógica. ${ }^{18}$

15 Hamlyn (1959) ilustra dicha circularidad usando como modelo el concepto de lenguaje, cuya aclaración, según argumenta, requiere la noción de referencia, la que a su vez se entiende por medio de la idea de 'función de ciertas expresiones'. El concepto de función de una expresión se explica apelando a la noción de 'sistema de reglas', y ésta presupone la noción misma de lenguaje (cfr. Hamlyn, 1959).

16 En 2.011 Wittgenstein afirma: "Es esencial al objeto poder ser la parte constitutiva de un estado de cosas"; de igual manera, en 2.014: "Los objetos contienen la posibilidad de todos los estados de cosas" y más adelante dice, en 2.0141: "La forma del objeto es la posibilidad de su ocurrencia en estados de cosas".

17 Por ejemplo, en 2.01 se afirma: "El estado de cosas es una conexión de objetos (cosas)" y en 2.03: "En el estado de cosas los objetos están unidos entre sí como los eslabones de una cadena". El hecho se define pues necesariamente a través de la noción misma de objeto.

18 Andreas Blank argumenta que la estructura conceptual del TLP puede pensarse "como una red de conceptos mutuamente interdependientes, en la cual ni conceptos lógicos ni ontológicos pueden ser definidos independientemente de todos los demás" (2007, p. 247). El problema es que el autor interpreta la circularidad como una circularidad entre ontología y lógica. Según Blank, solo a partir de tal concepción sistemática de la estructura conceptual del TLP es que puede comprenderse la analogía que el mismo Wittgenstein propone entre su propia comprensión acerca del método de la lógica y el método de la mecánica de Hertz. La comparación de Blank es interesante, pues resalta que así como la mecánica de Hertz constituye un intento por ofrecer una explicación unitaria del mundo físico, el TLP ofrece una aclaración de todo el espacio lógico a partir 


\section{El estatus especial de las proposiciones que contienen conceptos formales}

Wittgenstein afirma en el TLP que las proposiciones que contienen conceptos formales no tienen 'sentido', lo que quiere decir: que no representan ninguna situación o no describen ningún estado de cosas. Si dichas afirmaciones no representan ningún hecho o situación, por tanto, están al margen de la lógica de los valores de verdad. ${ }^{19}$ Esto es consecuencia de las características de dichos conceptos y de la finalidad para la cual se introducen en el TLP, que es la de aclarar los rasgos formales de distintas clases de signos. ${ }^{20}$ Kuusela enfatiza correctamente que si bien Wittgenstein niega la posibilidad de expresar la estructura de la proposición, y con ella del lenguaje, no niega que 'en algún sentido' es posible expresarla o hablar de ella, específicamente de dos maneras:

[...] en casos en los que las propiedades formales no se muestran fácilmente, sino que el lenguaje natural las obscurece o disimula, es posible que debamos hacer tales propiedades explícitas mediante la transformación de las expresiones, es decir, traduciendo las expresiones

del principio de la figuratividad del signo proposicional, formulando lo que Wittgenstein denominó la forma general de la proposición: 'las cosas están así y asá' o, en sus propias palabras: "las cosas se comportan de tal y tal modo" (TLP, 4.5). Sin embargo, es un error la escisión entre conceptos lógicos y conceptos ontológicos en el TLP. El TLP, como ha quedado sugerido, se ocupa específicamente de problemas de lógica, y los conceptos que allí se introducen están relacionados con el propio simbolismo del TLP; la idea misma de un 'concepto ontológico' está fuera de lugar, en el TLP solo se reconocen los conceptos formales, por un lado, y los conceptos propiamente dichos, por otro. Por otra parte, como bien se muestra en los estudios de Kuusela (2012, 2014), McGinn (2009), pero sobre todo en Mota $(2015,2018)$, es un error interpretar los conceptos formales como conceptos ontológicos. Bien afirma Mota que: "un concepto formal no es un objeto cuya existencia pueda ser descrita por una proposición (esto es, no es una cuestión ontológica, sino conceptual o gramatical, para Wittgenstein sinónimos)" (2015, p. 196), y la razón es que "la noción de categoría ontológica pertenece al lenguaje ontológico, mientras que los conceptos formales están presentes en otros usos debido a que son categorías lógicosintácticas (i.e. gramaticales) que no implican ontología en ningún sentido" (Mota, 2018, p. 325).

19 "Las proposiciones que las contienen no son figuras genuinas, es decir, no describen un estado de cosas que puede existir o no existir" (McGinn, 2001, p.31).

20 McGinn (2006) aclara que la idea de concepto formal está vinculada a la investigación wittgensteiniana sobre la naturaleza de las variables, esto es, a la investigación sobre cómo determinados símbolos simbolizan y, a su vez, esa investigación sobre las variables se enmarca en la investigación general sobre la lógica en el TLP. Según la autora, es interés de Wittgenstein en el Tractatus mostrar que la lógica se caracteriza por el recurso a variables y no, como llegó a pensar Russell, por la investigación de cierto tipo de objetos lógicos (2006, p. 166). Ver también Engelmann (2013, p. 133). 
en una notación lógicamente perspicua. Por lo tanto, un sentido en el que podemos, según Wittgenstein, hablar acerca de las propiedades formales o sintácticas es realizando un análisis lógico por medio de una notación simbólica. (Kuusela, 2012, p. 7)

Y la segunda:

Una segunda forma de hablar de la sintaxis [...] es introduciendo conceptos y principios sintácticos que gobiernen la notación lógicamente perspicua que se utiliza con el propósito del análisis lógico. Wittgenstein busca introducir tales conceptos y principios -por ejemplo, que las conectivas lógicas no representan objetos lógicos (TLP, 4.0312) o la noción de una forma general de la proposición- por medio de oraciones que, se espera, su lector llegue a reconocer como sinsentidos. (Kuusela, 2012, p. 7)

Estos dos últimos ejemplos: 'los conectores lógicos no representan objetos lógicos' y 'hay una forma general de la proposición' ilustran enunciados del TLP donde aparecen conceptos formales; en el primero está la noción de objeto, y en el segundo la noción de proposición, y ambos sirven como ejemplos de expresiones que carecen de valor de verdad, puesto que no describen ninguna situación, y que estipulan principios relativos al análisis lógico de la proposición. En este sentido es que debe entenderse el énfasis de Wittgenstein en que las proposiciones de la lógica no dicen nada: no hablan de ningún hecho del mundo, y lo mismo vale para las propias proposiciones del TLP que introducen, a la vez, conceptos formales y un lenguaje sígnico especial para el análisis. Por eso toda afirmación que contiene un concepto formal tiene la finalidad de indicar algo sobre la manera como una expresión simboliza. ${ }^{21}$

21 La distinción wittgensteiniana entre las proposiciones del TLP, que contienen conceptos formales y que están en función de aclarar algo sobre el simbolismo lógico y sobre la estructura de nuestras expresiones, y las proposiciones con sentido, está vinculada con la polémica en torno al propósito general que se anuncia en el prólogo del TLP: el de trazar límites; exactamente se habla allí de un límite que "sólo puede ser trazado en el lenguaje, y todo cuanto quede al otro lado del límite será simplemente un sinsentido". En 4.121 Wittgenstein hace explícita la naturaleza de dicho límite cuando traza la diferencia entre lo que el lenguaje dice y lo que en él se muestra. La parte más difícil del argumento es entender cómo se ha podido trazar dicho límite en el lenguaje, desde el propio lenguaje sin salirse de él. Con base en el numeral 4.122 se puede afirmar que para ello se ha debido introducir una manera de hablar 'inusual' con el fin de dar a entender lo que no se puede decir, esto es, aquello que solo puede mostrarse, las propiedades formales del lenguaje. Las afirmaciones del TLP constituyen también la herramienta para reconocer 


\title{
Disolución de las dificultades acerca del concepto caballo
}

\author{
Con base en la idea de concepto formal es posible ahora dar una
} respuesta clara al desafío que plantea el problema del 'concepto caballo'.22 Frege ya había advertido acerca de las dificultades con las que nos encontramos al intentar aclarar la diferencia entre concepto y objeto, esto es, cuando intentamos dar cuenta de las nociones fundamentales de la lógica, las cuales se necesitan para hablar sobre la estructura de nuestras expresiones y sobre las propiedades figurativas del signo proposicional. Esas dificultades se ponen de manifiesto

la diferencia entre las proposiciones con sentido, las que dicen cómo están las cosas, y las proposiciones sin sentido, las que no dicen nada sobre el mundo sino que ayudan a reconocer los rasgos formales de los símbolos en el entramado proposicional (cfr. Engelmann, 2013).

22 Una primera respuesta a esas dificultades que antecede a la solución del TLP la constituye la teoría de los tipos de Russell. En Los principios de la matemática (2010), Russell establece la distinción entre concepto y objeto introduciendo la noción de "término" la cual cubre por igual a objeto y concepto. Algunos términos solo pueden aparecer en la proposición como sujetos, nunca pueden ser predicados; éstos son llamados por Russell 'cosas'. Los otros términos pueden desempeñarse a la vez como sujetos o predicados, como es el caso de 'ser mortal' pues 'mortal' puede ser tanto predicado de Sócrates como sujeto de otro concepto, por ejemplo, del concepto 'cualidad de todo ser vivo'. Así se conserva la distinción entre concepto y objeto pues mientras los términos que son objetos solo pueden aparecer como sujetos gramaticales en una proposición, los términos que pueden aparecer como sujetos o predicados son los conceptos (cfr. Russell, 2010, p. 46). El análisis de Russell requiere sin embargo una restricción para prevenir la formación de absurdos y sinsentidos, ya que un concepto también puede ser sujeto gramatical, pero evidentemente una expresión como 'ser mortal es Sócrates' o 'cualidad (ser cualidad) es mortal' constituyen combinaciones ilegitimas de conceptos y objetos. Para evitar este tipo de sinsentidos se requiere una jerarquía de niveles de conceptos, y esto es lo que ofrece la teoría de los tipos que aparece por primera vez en el Apéndice B de Los principios de la matemática, con el fin de ofrecer una estratificación de conceptos que, una vez introducida, impide la confusión, no solo entre objeto y concepto, sino entre distintos tipos o clases de conceptos entre sí. La teoría de los tipos clasifica todo término bien sea como cosa o bien como función de algún 'nivel' u 'orden', de manera que al establecer la existencia de distintos tipos lógicos, introduce reglas y restricciones que impiden el surgimiento de expresiones absurdas o paradójicas de la siguiente manera: Russell distingue entre individuos, éstos constituyen el nivel 0; clases de individuos, éstos constituyen el nivel 1; luego, dice Russell: "The next type after classes of individuals consists of classes of classes of individuals" (2010, p. 536), y constituyen el nivel 2; y así sucesivamente. Según la teoría, una propiedad de nivel 1 solo puede ser atribuida o negada a individuos, esto es, al nivel 0 , pero no a propiedades de un nivel superior o del mismo nivel. Así 'ser caballo' se puede predicar de Bucéfalus, pero no puede predicarse de 'ser perro', concepto del mismo nivel, o de 'ser animal', concepto de nivel superior; una propiedad de nivel 2 solo puede ser atribuida o negada a propiedades de nivel 1, pero no a propiedades del mismo nivel o de un nivel superior, etcétera. Si se transgrede esta restricción aparecen secuencias de palabras como 'cualidad es mortal' que constituye un claro sinsentido, pero que se evita si cada concepto o propiedad de nivel n se atribuye solamente a conceptos del nivel $\mathrm{n}-1$. 
en las extrañas expresiones que el lógico articula en el intento por explicar esas categorías. Ejemplos paradigmáticos son las expresiones que Frege usa cuando intenta aclarar la naturaleza predicativa de todo concepto afirmando, por ejemplo, que "el concepto 'caballo' no es ningún concepto" o que "el concepto 'caballo' no es un objeto". Al parecer cada vez que intentemos decir algo sobre un concepto éste se convertirá ipso facto en objeto.

Todas estas dificultades, sin embargo, son vistas ahora desde una nueva perspectiva. En primer lugar, para Wittgenstein las dificultades que surgen a la hora de aclarar las propiedades lógicas de nuestro lenguaje están justificadas, ya que son inherentes a la pretensión de explicar lo que no constituye objeto alguno de descripción: la lógica de nuestro lenguaje, la cual solo se muestra en las propiedades estructurales de toda proposición con sentido.

Que una parte de una proposición funcione como concepto o como objeto es algo que se reconoce en el modo y manera como dicha parte simboliza en la proposición, esto es, en la forma como se comporta la expresión en el entramado proposicional. Así en una proposición como "Bucéfalus es un caballo", que el signo 'Bucéfalus' es el símbolo de un objeto se muestra en la proposición misma, y de igual forma, es manifiesto que el signo 'caballo' es parte del símbolo de un concepto, de '...es un caballo'. Igualmente en una proposición como "el caballo viene galopando" es claro que el signo 'caballo' es aquí el símbolo de un objeto y 'viene galopando' el símbolo de un concepto. Que la palabra caballo simboliza de forma y manera distinta en ambas proposiciones se reconoce en virtud del uso lógicosintáctico definido que en cada una tiene el signo 'caballo'. Por esta razón Wittgenstein advierte que: "El lenguaje no puede representar lo que en él se refleja. Lo que se expresa en el lenguaje no podemos expresarlo nosotros a través de él” (TLP, 4.121), es decir, lo que por su propia naturaleza se refleja en el uso del lenguaje no constituye objeto de descripción mediante proposiciones con sentido; es pues un absurdo hablar de las propiedades del 'concepto caballo', ya que ser concepto es un rasgo de ciertos símbolos, y no quedan propiedades específicas que se pueden describir: lo que pertenece a la lógica de la proposición se muestra en la misma proposición, las propiedades formales son rasgos lógico sintácticos que muestran los símbolos en su uso. De esta manera, comenta Linsky: 
En una notación lógica correcta, la oración comprometedora de Frege "El concepto caballo no es un concepto" no puede ser escrita. Que caballo es un concepto se muestra por medio de su uso, pero esto no se puede decir. Por lo tanto, el "grano de sal" ${ }^{\text {"3 }}$ que Frege reclama se requiere debido a una característica del lenguaje cotidiano que no se reproduce en ningún lenguaje construido de acuerdo con las reglas de la sintaxis lógica. (1992, p. 270)

Así pues Frege tiene razón cuando advierte que hay un inconveniente con nuestro lenguaje cuando intentamos hablar acerca de las propiedades formales de nuestras propias formas de expresión pero se equivoca cuando intenta dar expresión a dichas propiedades o enumerarlas. Por eso desde la propuesta del TLP el problema del 'concepto caballo' es solo aparente, y lo que produce ese aparente problema no es una insuficiencia de nuestro lenguaje ordinario para expresar ciertos hechos lingüísticos, éste solo aparece cuando se da una confusión entre conceptos propiamente dichos y conceptos formales. ${ }^{24}$

Wittgenstein pretende mostrar la diferencia entre concepto y objeto solo mediante una presentación perspicua de las reglas del puro simbolismo, a través de una notación lógica adecuada. ${ }^{25}$ Según la exigencia del TLP, se trata de una diferencia entre maneras de simbolizar los signos, de ahí el llamado de atención de Wittgenstein:

23 Linsky alude aquí a las Investigaciones lógicas donde Frege usa la expresión "tomar como 'un grano de sal"', la cual hace referencia a ser tomado con mucho cuidado o cautela, y que aplicado al caso de las expresiones quiere decir que deben ser tomadas en un sentido especial o fuera de lo común.

24 La solución de Russell mediante la teoría de los tipos no corre mejor suerte. Para Russell la diferencia entre concepto y objeto es en parte lógica y en parte metafísica. Objeto y concepto se diferencian porque son 'tipos' distintos, son expresiones que refieren a clases distintas de entidades; un objeto refiere una cosa, un concepto refiere una clase de un rango específico: clase de objetos, clase de clases de objetos, etcétera, teniéndose que admitir la existencia de una jerarquía de clases o de funciones para realizar de forma satisfactoria el análisis de las condiciones de significatividad de la proposición. La crítica fundamental de Wittgenstein a la solución de Russell radica en el rechazo a toda explicación lógica que hable acerca del significado de los signos, como la teoría de los tipos; "[...] el error de Russell se manifiesta en esto: que Russell, para establecer las reglas de los signos, ha tenido necesidad de hablar del significado del signo" (TLP, 3.331).

25 Podemos considerar con Engelmann que la notación lógica del TLP es expresión de la estructura a priori del lenguaje, por medio de reglas también a priori. Que las reglas de la notación lógica sean a priori quiere decir que tales reglas "tienen que comprenderse por sí mismas, con sólo saber cómo designa cada signo" (TLP, 3.334), y que expresen la estructura a priori del lenguaje es posible porque las reglas de la notación lógica se expresan únicamente haciendo uso de variables. 
La sintaxis lógica no permite que el significado de un signo juegue en ella papel alguno; tiene que poder ser establecida sin mentar el significado de un signo; ha de presuponer sólo la descripción de las expresiones. (TLP, 3.33) $)^{26}$

De esta manera, que 'caballo' sea un concepto no radica en propiedad alguna de lo que el signo designa sino en las propiedades formales o, lo que es lo mismo, en el uso lógico sintáctico de la expresión caballo. Cuando Wittgenstein habla de la "descripción de la expresión" se refiere a la aclaración de las propiedades lógicas del símbolo que, como sugiere Kuusela (2012), es posible a través de una notación lógica perspicua y de las proposiciones del TLP que acompañan el recurso a dicha notación. ${ }^{27}$

Engelmann lo expresa de igual manera cuando dice que las afirmaciones del TLP están en función de aclarar la notación lógica del TLP, lo que equivale a decir que sus proposiciones nos conducen a ver con claridad las reglas implícitas de nuestro lenguaje ordinario, que en un cierto sentido dominamos puesto que somos hablantes competentes de nuestros propios lenguajes naturales, pero que no podemos expresar sin la ayuda de un mecanismo adecuado, una notación lógica perspicua como la que pretende ofrecer el TLP. Y como bien aclara Engelmann, dado que dicha notación debe hablar por sí misma las afirmaciones del TLP se tornan superfluas una vez que comprendemos la notación lógica, "ellas simplemente lo llevan a uno a ver explícitamente en la notación lo que ya uno conoce implícitamente" (Engelmann, 2013, p. 134).

Wittgenstein quiere pues librarnos de la confusión de creer que una investigación acerca de las propiedades lógicas del lenguaje es una investigación sobre lo que los símbolos significan, y no una investigación sobre cómo los símbolos simbolizan, es decir, sobre cómo se usan los símbolos en una proposición con sentido

26 McGinn (2006) habla de 'modes of signification' para referirse a eso que desde el TLP es la meta del análisis: "los conceptos formales se ocupan de los modos de significación y esto es algo que se puede establecer 'sin mencionar el significado del signo: solo se presupone la descripción de la expresión' (TLP, 3.33)” (McGinn, 2006, p. 184).

27 De forma semejante en McGinn: "Los conceptos formales -el concepto de un nombre, un objeto, una función, una proposición, etc.- son expresiones que pretenden describir la categoría lógico-sintáctica de una expresión, es decir, describir lo que es esencial para el sentido de un signo" (2001, p. 31). 


\section{Referencias}

Blank, A. (2007). Material Points and Formal Concepts in the Early Wittgenstein. Canadian Journal Of Philosophy, 37(2), 245-262. Recuperado de https://bit.ly/2yh9y3Z

Dean, K. (2007). The Concept "Horse" Paradoxand Wittgensteinian Conceptual Investigations: A Prolegomenon to Philosophical Investigations. Farnham: Ashgate Publishing Ltd.

Engelmann, M. (2013). Wittgenstein's Philosophical Development: Phenomenology, Grammar, Method, and the Anthropological View. New York: Palgrave Macmillan.

Frege, G. (1972 [1879]). Conceptografía (Begriffsschritt, eine der arithmetischen nachgebildete Formelsprache des reinen Denkens, H. Padilla, Trad.). México: Universidad Autónoma de México.

Frege, G. (1974 [1892]). Escritos lógico semánticos (C. R. Luis y C. Pereda, Trads.). Madrid: Técnos.

Hamlyn, D. (1959). Categories, Formal Concepts and Metaphysics. Philosophy, 34(129), 111-124. doi: https://doi.org/10.1017/S003181910 0047458

Hacker, P. (2001). Wittgenstein: Connections and Controversies. Oxford: Clarendon Press.

Ishiguro, H. (1969). Use and Reference of Names. In P. Winch (Ed.), Studies in the Philosophy of Wittgenstein (pp. 20-50). London: Routledge.

Kenny, A. (1995). Frege: An Introduction to the Founder of Modern Analytic Philosophy. London: Blackwell Publishers

Kienzler, W. (2011). Reading the Tractatus from the Beginning: How to say everything clearly in three words (Jena, 2008, slightly revised version July 2011). Recuperado de https://bit.ly/2lf7dxm

Koethe, J. (1996). The continuity of Wittgenstein's thought. Ithaca: Cornell University Press.

Kuusela, O. (2012). Carnap and the Tractatus' philosophy of logic. Journal for the History of Analytical Philosophy, 1(3), 1-25. doi: https://doi. org/10.4148/jhap.v1i3.1334

Kuusela, O. (2014). The Method of Language-Games as a Method of Logic. Philosophical Topics, 42(2), 129-160. Recuperado de https://bit. ly/2yhaj3u

Linsky, L. (1992). The Unity of the Proposition. Journal of the History of Philosophy, 30(2), 243-273. Recuperado de https://bit.ly/2LZ1zeb 
McGinn, M. (2001). Saying and Showing and the Continuity of Wittgenstein's Thought. The Harvard Review of Philosophy, (IX), 2436. Recuperado de https://bit.ly/2JTX8Aq

McGinn, M. (2006). Elucidating the Tractatus: Wittgenstein's Early Philosophy of Logic and Language. Oxford: Oxford University Press.

McGinn, M. (2009). Wittgenstein and internal relations. European Journal of Philosophy, 18, 495-509. doi: https://doi.org/10.1111/j.14680378.2009.00359.x

Mota, S. (2015). Wittgenstein en torno a los conceptos. Análisis. Revista de Investigación filosófica, 2, 195-219. doi: https://doi.org/10.26754/ojs_ arif/a.rif.201511119

Mota, S. (2018). ¿Son los conceptos formales (o lógicos) categorías ontológicas? Tópicos. Revista de Filosofía, 54, 301-331. doi: http://dx.doi. org/10.21555/top.v0i54.905

Phillips, D. (2007). Complete analysis and clarificatory analysis in Wittgenstein's Tractatus. In M. Beaney (Ed.), The Analytic Turn, Analysis in Early Analytic Philosophy and Phenomenology (pp. 164-177). New York: Routledge.

Russell, B. (2010 [1903]). Principles of Mathematics. London: Routledge Clasics.

Wittgenstein, L. (1979). Cartas a Russell, Keynes y Moore. Madrid: Taurus.

Wittgenstein, L. (2009). Diarios secretos. Madrid: Alianza.

Wittgenstein, L. (2009 [1922]). Tractatus Logico-Philosophicus (J. Muñoz Veiga e I. Reguera Pérez, Trads.). Madrid: Gredos. 